\title{
THE EFFECT ON THE HUMAN THIRD HEART SOUND OF VARIATIONS IN THE RATE OF FILLING OF THE HEART
}

\author{
BY \\ A. W. SLOAN AND MARY WISHART \\ From the Institute of Physiology, University of Glasgow \\ Received May 29, 1952
}

The first descriptions of the physiological third heart sound (Gibson, 1907; Hirschfelder, 1907) related its occurrence to the presence of a wave in the venous pulse immediately following the $\mathbf{V}$ wave. Thayer (1908) showed that the sound occurs simultaneously with the downstroke of the $\mathrm{V}$ wave or the upstroke of the wave that immediately follows; this corresponds to the period in the cardiac cycle when the rate of filling of the ventricles is maximal, just after the opening of the atrio-ventricular valves. Because of its time of occurrence the third heart sound has been called the " rapid-filling" sound.

The physiological third heart sound is best heard at, or a short distance medial to, the cardiac apex, with the subject recumbent and lying on the left side. On auscultation it seems louder if the rate of venous return of blood to the heart is increased by moderate exercise (Gallavardin, 1912), by raising the patient's arms and legs (Thayer, 1909), or by pressure on the abdomen (Gibson, 1907).

Observers who attempted to record the third heart sound by phonocardiography after exercise have differed in their findings. Bridgman (1915) found an increase in the sound deflections immediately after exercise, whereas Mannheimer (1940) found a diminution.

The improved electronic techniques employed in modern phonocardiography offer an opportunity to demonstrate objectively any alterations in loudness of the heart sounds with varying rates of venous inflow of blood. Linear, stethoscopic, and logarithmic phonocardiograms may be recorded as recommended by Rappaport and Sprague (1942). The deflections on the linear records vary in size with the amplitude of the original vibrations. The deflections on the logarithmic records vary with the loudness of the original sounds as heard by the human ear.

\section{METHOD}

The subjects selected for the investigation were 16 healthy young adults, each of whom showed an obvious third heart sound on the logarithmic phonocardiogram. The group consisted of 10 men and 6 women, and their ages ranged from 16 to 24 years.

The electronic phonocardiograph employed to record the heart sounds is fully described elsewhere (Campbell, Sloan, and Andrew, 1952). The jugular phlebogram was used as the reference tracing for the phonocardiogram since the physiological third heart sound can be distinguished from other diastolic sounds by its relationship to the $V$ wave of the phlebogram. An electrocardiogram (lead II) was also recorded from each subject to confirm the interpretation of the phlebogram.

Five recordings were taken from each subject. On each one the amplitude of deflection for each sound was measured over five cardiac cycles and the average amplitude calculated.

The first two records were preliminary controls, one with the cardiogram, and the other with the phlebogram as reference tracing; they were taken after the subject had been lying down for approximately five minutes. Sphygmomanometer cuffs were then applied to both thighs and both arms 
and were inflated to the diastolic blood pressure of the individual. According to McMichael and Sharpey-Schafer (1944) interruption of the venous return from the limbs by this method produces a considerable fall in the right atrial pressure. The sphygmomanometer cuffs were rapidly inflated by connecting them through a stopcock to a reservoir of compressed air; the cuffs were maintained at diastolic blood pressure for five minutes, at the end of which time a further recording was taken. The pressure was then released and, after allowing a further five minutes for the circulation to return to normal, another control record was taken. The subject then performed a cycling movement of the legs in the air for two minutes, while remaining supine, and a final record was taken immediately on completion of this exercise.

The chest-piece of the stethoscope leading to the microphone was firmly held over the cardiac apex by a rubber band round the chest and remained in position throughout each observation. In two cases no valid record was obtained after exercise due to shifting of the chest-piece during the muscular movement. The gain of the amplifier was constant during each observation but it was of necessity altered for different subjects owing to the wide individual variation in intensity of the heart sounds. Consequently the alterations in amplitude of the phonocardiogram deflections were comparable within each individual experiment but were not comparable from subject to subject. The changes in amplitude were therefore determined in terms of percentage increase or decrease.

\section{RESULTS}

The records obtained from one of the subjects are shown in the Figure. Venous occlusion is seen not to affect the first and second sounds but the third sound is almost abolished. Exercise results in a considerable increase in amplitude of both the first and the third sounds.

With venous occlusion the third sound deflections were either abolished or diminished in every subject. The individual changes were relatively uniform, the extreme values being -50 per cent and -100 per cent. The effect on the first and second sounds was negligible, the individual changes being small and variable.

After exercise the third sound deflections were increased in amplitude in 10 of the 14 subjects, the individual changes varying from +10 per cent to +200 per cent. Four subjects showed no alteration in the amplitude of the third sound. The first sound was increased in amplitude in every subject. The second sound showed a variable response; in the majority of cases there was a small increase. The mean reduction in amplitude of the third sound deflections with venous occlusion and the mean increases of first and third sound deflections after exercise were statistically highly significant $(\mathrm{P}<0.01)$.

There was a negligible change in heart rate with venous occlusion of the limbs. The rate was increased with exercise in every case but one. The individual alterations in the heart rate with exercise did not correspond to the individual variations in the response of the third heart sound.

\section{Discussion}

Of the three normal heart sounds, only the third is consistently diminished by reduction in the rate of filling of the heart.

An increase in the rate of filling of the heart increases the amplitude of the vibrations of the first and third sounds but the effect on the third sound is not so consistent as that caused by reducing the venous return. This might be expected from the contradictory findings of Bridgman (1915) and Mannheimer (1940). Since there is no correlation between the rate of the heart and the amplitude of the deflections of the third heart sound, the failure of the sound to increase after exercise in some cases cannot be explained by a reduced inflow per beat due to tachycardia. It seems, therefore, that other circulatory changes associated with muscular exercise, in addition to the rate of inflow of blood to the ventricles, must influence the production of the third heart sound. 


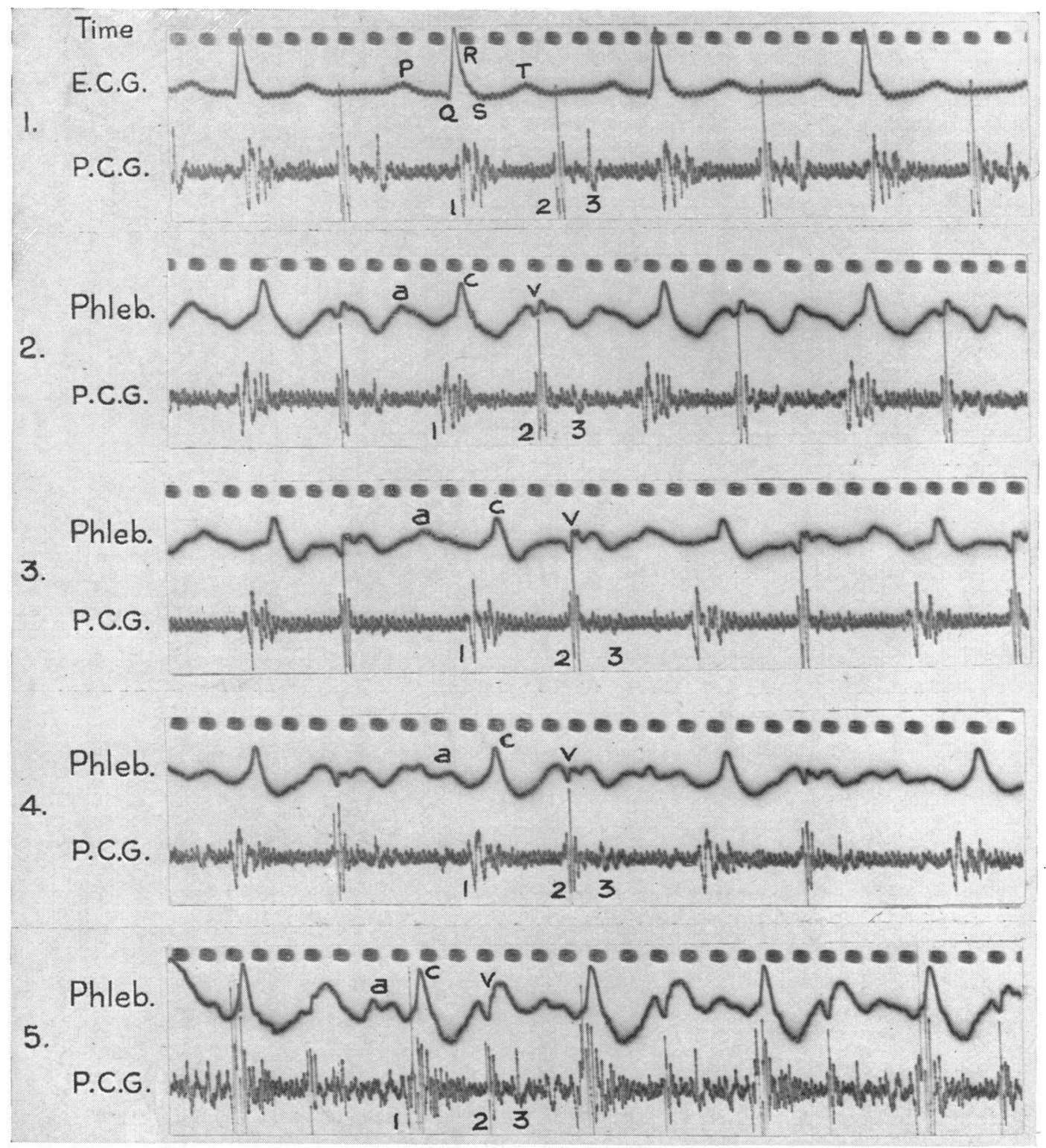

Fig.-Set of records from one subject. 1 and 2, preliminary controls; 3, with venous occlusion; 4, control after venous occlusion; 5, after exercise. E.C.G.=electrocardiogram (lead II); Phleb.=jugular phlebogram; P.C.G. $=$ logarithmic phonocardiogram. Time marking in $1 / 10 \mathrm{sec}$.

The balance of evidence seems to favour the relationship of the third heart sound to the rate. of filling of the heart and to justify the use of the term, " rapid-filling " sound.

\section{SUMMARY}

Logarithmic phonocardiography on 16 human subjects showed that deflections due to the third heart sound were reduced in amplitude in every case when the venous return from the limbs was obstructed. 
In 10 out of 14 subjects the third sound deflections were increased in amplitude after muscular exercise: in the remaining 4 the deflections were unchanged.

Changes in the first and second sounds were less consistent.

Our thanks are due to Professor R. C. Garry for advice and criticism and to Mr. A. C. Bain for technical assistance Expenses were defrayed by a grant from the Rankin Medical Research Fund of the University of Glasgow.

\section{REFERENCES}

Bridgman, E. W. (1915). Heart, 6, 41.

Campbell, F. W., Sloan, A. W., and Andrew, A. M. (1952). Brit. Heart J., 14, 271.

Gallavardin, L. (1912). Arch. Mal. Caur, 5, 776.

Gibson, A. G. (1907). Lancet, 2, 1380.

Hirschfelder, A. D. (1907). Johns Hopkins Hosp. Bull., 18, 265.

McMichael, J., and Sharpey-Schafer, E. P. (1944). Brit. Heart J., 6, 33.

Mannheimer, E. (1940). Acta padiatr., Stockh., 28; Supp. 2.

Rappaport, M. B., and Sprague, H. B. (1942). Amer. Heart J., 23, 591.

Thayer, W. S. (1908). Boston med. surg. J., 158, 713.

(1909). Arch. intern. Med., 4, 297. 